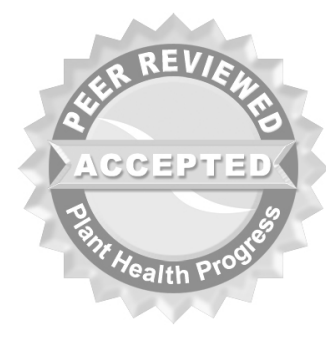

(C) 2012 Plant Management Network.

Accepted for publication 12 June 2012. Published 14 July 2012.

\title{
Susceptibility of Arabidopsis Ecotypes to Infection by Iris yellow spot virus
}

\author{
Khalid Naveed and Hanu R. Pappu, Department of Plant Pathology, \\ Washington State University, Pullman, WA 99164 \\ Corresponding author: Hanu R. Pappu. hrp@wsu.edu
}

Naveed, K., and Pappu, H. R. 2012. Susceptibility of Arabidopsis ecotypes to infection by Iris yellow spot virus. Online. Plant Health Progress doi:10.1094/PHP-2012-0714-01-RS.

\begin{abstract}
Iris yellow spot virus (IYSV; genus Tospovirus, family Bunyaviridae) is a serious constraint for onion production in the United States and several other parts of the world. A few experimental indicator hosts were reported for IYSV but there is no report of infection of Arabidopsis by IYSV. Establishing IYSV infection in onion by mechanical inoculation has so far proven to be inefficient resulting in numerous escapes. Using Arabidopsis thaliana as a host for studies on the biology and tospovirus-host interactions has an advantage of the plant's small genome size, short life cycle, and a genome that has been sequenced. Several ecotypes were evaluated for their susceptibility to IYSV and identified some where IYSV produces localized infection. Together with wild type and various mutants, the IYSVArabidopsis system could be useful for studying host-tospovirus interactions.
\end{abstract}

\section{Introduction}

Iris yellow spot virus (IYSV; genus Tospovirus, family Bunyaviridae) is a serious constraint for onion production in the United States and several other parts of the world (3,7). A few experimental indicator hosts were reported for IYSV (1) but there is no report of infection of Arabidopsis by IYSV. Establishing IYSV infection in onion by mechanical inoculation has so far proven to be inefficient resulting in numerous escapes. Using Arabidopsis thaliana as a host for studies on the biology and tospovirus-host interactions has an advantage of the plant's small genome size, short life cycle, and a genome that has been sequenced (8). Tomato spotted wilt virus, the type member of the genus Tospovirus, can infect Arabidopsis $(2,4,5)$. To investigate if Arabidopsis could be used as a model plant for studying IYSV-host interactions, we evaluated the response of different Arabidopsis ecotypes to IYSV infection in a controlled environment.

Seeds of three Arabidopsis ecotypes, Columbia wild type (Col-o), Landsberg erecta, and Wassilewskija, were sown in Murashige-Skoog medium containing $0.8 \%$ agar under white light at $23^{\circ} \mathrm{C}$. One-week-old seedlings were transplanted in small $28-\times 55-\mathrm{cm}$ plastic trays with 36 inserts having SunShine potting mixture LC1. Plants were shifted from incubator to growth chamber with a temperature of $18^{\circ} \mathrm{C}$ and a day-night cycle of $12 \mathrm{~h}$ with $160 \mu \mathrm{mol} / \mathrm{cm}^{2}$ light intensity.

IYSV-infected datura leaves were ground in chilled o.1 $\mathrm{M}$ phosphate buffer (pH 7.0) having 4\% $\beta$-mercaptoethanol and the homogenate was used for inoculation of healthy Arabidopsis plants. Four to five fully expanded leaves of plants were dusted with carborundum powder (600 mesh) and the inoculum was applied to leaves with cotton buds. In each independent experiment, twenty plants of each ecotype were inoculated with IYSV. Mock-inoculated Arabidopsis plants (Fig. 1A) consisted of plants inoculated with buffer only and maintained in the same growth chamber. Mock-inoculated controls were tested before mock-inoculation and during the course of the experiment using RT-PCR for the nucleocapsid protein gene $(\mathrm{N})$ of IYSV to ensure that they remained free of IYSV infection. In each independent experiment, five mock-inoculated controls were included. Each independent experiment was repeated four times. 
Leaves of inoculated plants started showing wilting and purple discoloration two weeks-post inoculation. With increasing number of days-post inoculation, purple discoloration and wilting became more severe resulting in dying of leaves. Some of the plants also showed characteristic diamond-shaped lesions which is a typical symptom of IYSV infection in onion (3). Infected plants also showed chlorotic spots and yellowing of leaves (Fig. 1B).

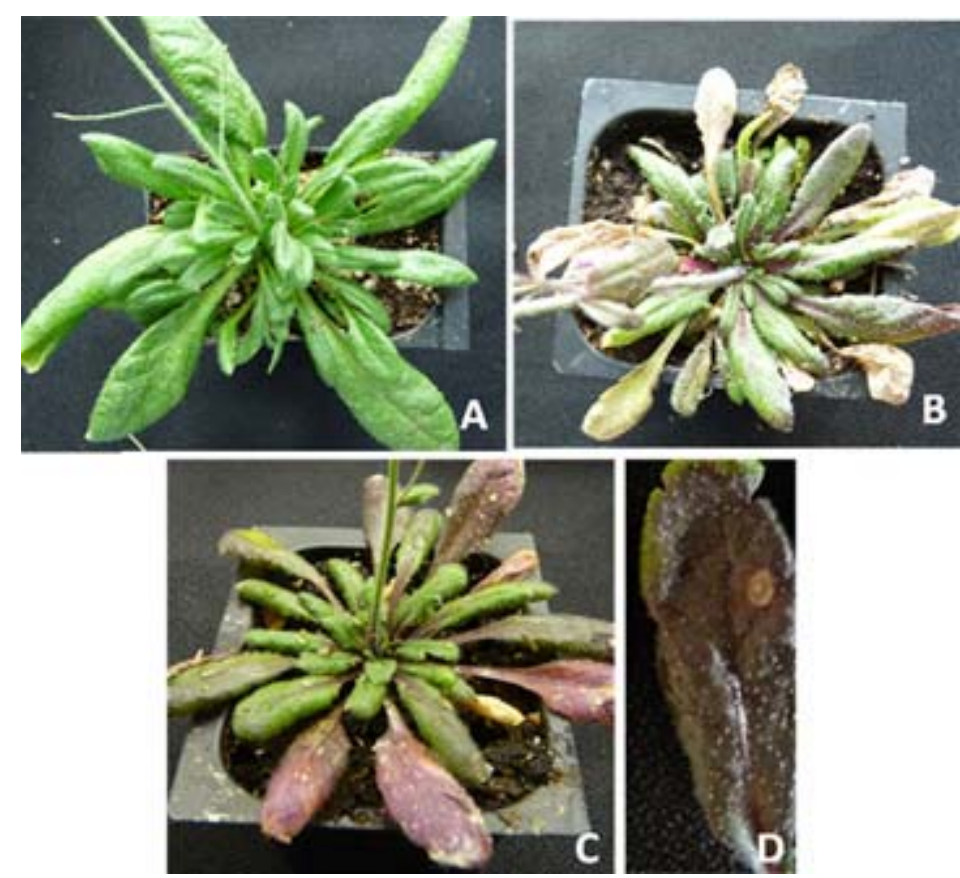

Fig. 1. Arabidopsis plants showing symptoms of Iris yellow spot virus (IYSV) and Tomato spotted wilt virus (TSWV) infection: (A) healthy Arabidopsis plant; (B) IYSV-infected Arabidopsis plant showing yellowing, wilting, and purple discoloration of leaves; (C) TSWV-infected Arabidopsis plant showing yellowing, wilting, and purple discoloration of leaves; (D) Arabidopsis leaf with IYSV-induced lesion.

Leaves showing purple discoloration and wilting were collected 20 days postinoculation and tested for IYSV infection by ELISA following kit instructions (Agdia Inc., Elkhart, IN) and RT-PCR. Using ELISA, leaves from 14 inoculated plants of each ecotype were tested in duplicate wells and absorbance was recorded at $\mathrm{A}_{405} \mathrm{~nm}$. For Columbia ecotype, 8 out of 14 samples were positive by ELISA, and for Wassilewskija ecotype, 8 out of 16 samples were IYSV positive. None of the Landsberg erecta plants turned out positive to IYSV infection in ELISA. The average ELISA values for Columbia ecotype were $0.37 \pm 0.13$ for IYSV-inoculated plants in comparison to the healthy plants which gave an average of $0.12 \pm 0.0049$ absorbance value. The average ELISA value of the duplicate wells for positive IYSV-infected Datura stramonium control was $1.03 \pm 0.108$. For Wassilewskija ecotype, the ELISA values for IYSV-infected plants were $1.113 \pm 0.52$ in comparison to the healthy controls with an average ELISA reading of $0.199 \pm 0.0035$. Younger, uninoculated leaves from all ELISApositive plants of each ecotype were tested for systemic infection with IYSV by ELISA. None of the plants showed systemic infection.

Absence of systemic infection was further verified by RT-PCR by taking one uninoculated, younger leaf from six IYSV ELISA positive plants of each ecotype. In RT-PCR, primers specific to the nucleocapsid (N) gene coded by the small RNA (S-RNA) of IYSV were used (6). The forward and reverse primers, 5 ' CTCTTAAACACATTTAACAAGCA 3' and 5' TAAAACAAACATTCAAACAA 3', respectively, amplified a $1100 \mathrm{nt}$ fragment of the N gene (Fig. 2). PCR conditions consisted of an initial heating at $94^{\circ} \mathrm{C}$ for $1 \mathrm{~min}$, followed by 30 cycles (denaturation at $94^{\circ} \mathrm{C}$ for $10 \mathrm{sec}$, annealing at $55^{\circ} \mathrm{C}$ for $10 \mathrm{sec}$, and initial extension at $72^{\circ} \mathrm{C}$ for $1 \mathrm{~min}$ and $10 \mathrm{sec}$ ), and a final extension step at $72^{\circ} \mathrm{C}$ for 
7 min. Results of RT-PCR showed that the virus did not spread systemically to younger, uninoculated leaves. On the basis of ELISA results and RT-PCR, it was concluded that IYSV was restricted to inoculated leaves.

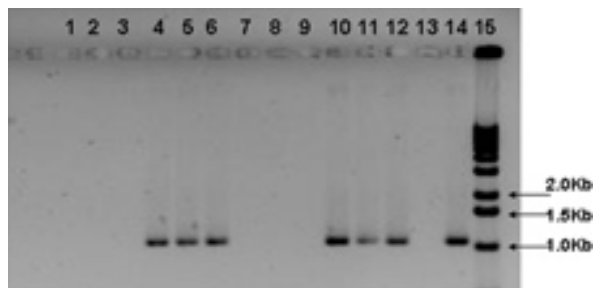

Fig. 2. Detection of Iris yellow spot virus (IYSV) in inoculated and uninoculated leaves of Columbia and Wassilewskija by reverse transcription polymerase chain reaction. Uninoculated leaves (lanes 1 to 3 ) and inoculated leaves from Wassilewskija (lanes 4 to 6 ); uninoculated leaves (lanes 7 to 9) and inoculated leaves from Columbia (lanes 10 to 12$)$. Template-free negative control (lane 13); IYSV infected Datura as positive control (lane 14); O' Gene Ruler 1Kb DNA ladder (Fermentas, Glen Burnie, MD) (lane 15). Arrow indicates amplicon of expected size for IYSV $\mathrm{N}$ gene by RT-PCR.

These findings indicate that Arabidopsis ecotypes Columbia and Wassilewskija are susceptible to infection by IYSV by producing localized infection. However, the virus failed to become systemic under the conditions used. To ensure that the conditions that were used are amenable for the inoculated plants to sustain virus infection, twenty plants of Columbia ecotype were inoculated with Tomato spotted wilt virus (TSWV) as it is reported to cause systemic infection in Arabidopsis. As reported earlier, TSWV produced systemic infection in Columbia ecotype (Fig. 1C) as demonstrated by ELISA. The ELISA values for systemic infection of Columbia with TSWV ranged from $1.48 \pm 0.85$ as compared to the healthy controls which gave an average $0.125 \pm 0.0056$ ELISA value. This showed that the conditions we used were suitable for the systemic infection of another tospovirus, TSWV, in Arabidopsis.

Mechanical inoculation of onion with IYSV is inefficient and there is a need to identify other experimental hosts to use for IYSV-host interactions. Our results show that Arabidopsis is susceptible to infection by IYSV and could be a useful host to study the virus-host interactions. Previous reports of tospovirus infection of Arabidopsis include Groundnut ring spot virus (GRSV) and Tomato spotted wilt virus (TSWV), which produced systemic infection (2). Interestingly in the case of IYSV, out of the three ecotypes tested, two ecotypes of Arabidopsis showed localized infection by producing symptoms on inoculated leaves and infection was confined to inoculated leaves only. The differential response of Arabidopsis ecotypes to tospoviruses indicates that different host and/or virus factors may be involved in the outcome of interaction between Arabidopsis and tospovirus. It remains to be seen if other ecotypes of Arabidopsis produce systemic infection to the tested isolate or other isolates of IYSV. Screening of Arabidopsis mutants for their enhanced resistance/susceptibility to IYSV infection could provide insights into the virus infection mechanism. As IYSV produced local infection that contrasts with the systemic infection of TSWV and GRSV, future studies can be conducted to unravel the basis for this localized infection of IYSV in Arabidopsis in comparison to systemic infection of GRSV and TSWV. 


\section{Acknowledgments}

The study was funded in part by a grant from the USDA NIFA Specialty

Crops Research Initiative (SCRI) \#2010-01193.

PPNS \#0601, Department of Plant Pathology, Department of Plant

Pathology, College of Agricultural, Human and Natural Resource Sciences, Agricultural Research Center, Project \#WNPO 0545, Washington State University, Pullman, WA 99164-6430, USA.

\section{Literature Cited}

1. Bag, S., and Pappu, H. R. 2009. Symptomatology of Iris yellow spot virus in selected indicator hosts. Online. Plant Health Progress doi:10.1094/PHP-2009-0824-01BR..

2. Elizabete, S. C., Lilian, S. T. C., Pollyanna, F. C., Barbara, S., Antonio, C. A., and Quirino, B. F. 2005. Evaluation of Arabidopsis thaliana response to infection by Tomato spotted wilt virus and Groundnut ring spot virus. Fitopatol. Bras. 31:1.

3. Gent, D., du Toit, L. J., Fichtner, S. F., Mohan, S. K., Pappu, H. R., and Schwartz, H. F. 2006. Iris yellow spot virus: An emerging threat to onion bulb and seed production. Plant Dis. 90:1468-1480.

4. German T. L., Scott, A., Andy, W., Kathryn, E. R., William, R. K., and Willis, D. K. 1995. Infection of Arabidopsis thaliana ecotype Columbia by tomato spotted wilt virus. Plant Mole. Biol. Reporter 13:110-117.

5. Edwards, H. 1999. Evaluating resistance to Tomato spotted wilt virus in Arabidopsis thatiana. J. Undergrad. Res. 1:1-4.

6. Pappu, H. R., du Toit, L. J., Schwartz, H. F., and Mohan, S. K. 2006. Sequence diversity of the Nucleoprotein gene of Iris yellow spot virus (genus Tospovirus, family Bunyaviridae) isolates from the western region of the United States. Arch. Virol. 151:1015-1023.

7. Pappu, H. R., Jones, R. A. C., and Jain, R. K. 2009. Global status of tospovirus epidemics in diverse cropping systems: Successes gained and challenges ahead. Virus Res. 141:219-236.

8. The Arabidopsis Genome Initiative. 2000. Analysis of the genome sequence of the flowering plant Arabidopsis thaliana. Nature 408:796-815. 